\title{
An Enquiry into VR Interface Design for Medical Training: VR Augmented Anatomy Tutorials for Breast Cancer
}

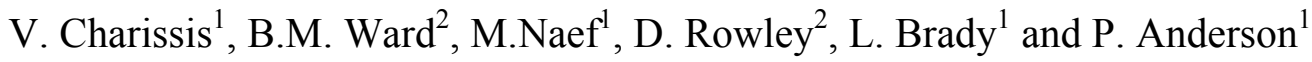 \\ ${ }^{1}$ University of Glasgow / Glasgow School of Art \\ Digital Design Studio, Glasgow, UK \\ ${ }^{2}$ Royal College of Surgeons, Edinburgh, \\ (v.charissis@gsa.ac.uk, ben.ward@ed.ac.uk)
}

\begin{abstract}
This paper presents an initial study exploring and evaluating a novel, accessible and user-centred interface developed for a VR Medical training environment. In particular, the proposed system facilitates a detailed 3D information exchange, with the aim of improving the user's internal 3D understanding and visualisation of complex anatomical inter-relationships. In order to evaluate the effectiveness of the proposed VR teaching method we developed a female 3D model under the guidance of Consultant Breast surgeons with particular emphasis given on the axilla section. In turn we commenced a comparative study between PBL tutorials augmented with VR and the contemporary teaching techniques involving twelve participants. Overall the paper outlines the development process of the proposed VR Medical Training environment, discusses the results from the comparative study, and offers suggestions for further research and a tentative plan for future work.
\end{abstract}

\section{KEYWORDS}

VR Interface, Medical Visualisation, Anatomy, Medical Education, HCI, Breast Cancer, Surgical Training, Haptics.

\section{INTRODUCTION}

This paper presents an initial study exploring and evaluating a novel, accessible and user centred interface developed for a VR Medical training environment. In particular, the proposed system should facilitate detailed 3D information exchange, with the aim of improving the user's internal 3D understanding and visualisation of complex anatomical inter-relationships. Evidently, recent studies advocate the benefits of VR training methods, which can provide beneficial outcomes by shortening the learning curve [1]. Furthermore complicated anatomical issues can be visualised intuitively by a VR interface, which does not have any physical constrains [2]. Hence it is possible for the user to interact freely with a volumetric 3D model, selecting their own number of infinite viewpoints, a factor suggested to be central in spatial anatomical understanding [3]. In addition the importance of facilitating a balanced deep learning approach including visualisation skills has been highlighted [4].

It is recognised that undergraduate anatomical training has changed in recent years. The availability of cadavers to undergraduates has significantly decreased. In recent years, increasing student numbers and pressures from a growing undergraduate curriculum has resulted in a decimation of students' exposure to cadaveric material and a reduction in total anatomical teaching [5]. It is suggested that current

The Engineering Reality of Virtual Reality 2008, edited by lan E. McDowall, Margaret Dolinsky, Proc. of SPIE-IS\&T Electronic Imaging, SPIE Vol. 6804, 680404, (C 2008 SPIE-IS\&T · 0277-786X/08/\$18 
medical and surgical trainees require anatomical resources that are convenient and adaptable to desired educational content and techniques $[6,7]$.

\section{EDUCATIONAL MODEL}

Recently there has been increasing interest in contextual situated learning in all disciplines, especially those requiring the application of integrated complex knowledge. A natural extension of the constructivist and situated approaches is the activity-based approach [8], where instructors frame the desired outcomes in terms of knowledge, attitude, skill and behaviours and design the learning activity to facilitate the active internalisation of different types of knowledge and meta-cognitive processes.

Medical Educationalists have become increasingly interested in the inherent opportunities that these techniques offer, in particular when knowledge and skills must be demonstrated perfectly in high stakes situations and challenging social environments. To this end there have been attempts to produce 'thickly authentic' interactive learning techniques to allow knowledge, skills, behaviours and reasoning to be learned in a clinically useful and therefore relevant manner [9]. These simulations also allow the student to fail safely, gaining from the learning experiences of failure with no risk to the patient. This approach has been mirrored by computer educational theorists, describing the role 'epistemic gaming' [10] i.e.: highly interactive problem based resources placed firmly in a realistic professional context. These two approaches highlight a growing area of interest in medical training; structured, contextualised learning in a virtual or 'real-world' environment that mirrors contemporary or professional challenges.

However within medical educational literature there remains doubt regarding the educational benefits of 3D visualisation (stereoscopic) vs. 2D applications in teaching spatial anatomy and operative procedure. Existing publications regarding the advantages of 3D display and rendering look mainly at rotational 2D representations of 3D anatomy in core undergraduate teaching. Stereoscopic projection has been demonstrated repeatedly to improve learning outcomes in training outside medicine [11, 12]. Additionally the specific advantages of free interaction with rotational models when learning complex 3D anatomy has been identified [3]. Also the importance of understanding the role of student spatialskills has been identified [13]. Currently many described studies exist in isolation from existing educational approaches and are de-contextualised from practical implementation. It was an aim of the inquiry to look at the use of 3D visualization as a resource for student-centred, situated, activity-based and activity-centred design [14] that could be implemented in surgical education.

Adhering to the aforementioned approaches we decided to evaluate the desired learning outcomes for surgical trainees in the subject domain of the clinical and operative management of advanced breast cancer. In association with the Edinburgh Breast Unit and utilising the transitional intercollegiate Membership of the Royal College of Surgeons (MRCS) examination we illuminated the key knowledge required. This was identified in terms of applied basic science, clinical principles and knowledge of operative procedures and protocol. In addition we identified the key skills and processes involved in sentinel node biopsy and the operative approach to axillary clearance. Additionally we developed a series of common case vignettes to create subject interaction and therefore explore reasoning. These principle goals were then mapped to situated activities which formed the basis of the design requirements for the structured tutorials and the semi-immersive VR application.

The primary goal was to place the student in a situation in which they experienced 'thickly authentic', situated learning in the context of a tutorial with a surgical trainer. A framework for this was produced and the VR system described below designed to meet these goals. It was important that an integrated approach was taken in all aspects of design, in order that we could create a design method embracing 
surgical educational needs, accurate anatomical representation and seem-less information transfer within the described educational model.

\section{VR REQUIREMENTS}

The aforementioned contemporary issues in medical training fuelled the development of the VR environment, which was based in the existing Digital Design Studio VR facilities. The particular system is developed around a Fakespace Immersive Workbench and entails real-time visualisation, gesture interaction with tactile feedback (CyberTouch ${ }^{\mathrm{TM}}$ sensored glove) and 3D sound as depicted in figure $1(a, b)$. Its semi-immersive design is particularly valuable in assisting with small group tutorial requirements.

Although the particular Workbench was initially developed for automotive engineering evaluation [15] it was found that the table-design of the installation offered an intriguingly similar position and dimensions to an operation table or an examination bed. Hence the existing VR and haptic technology was repositioned in order to enhance this feeling, replicating as much as possible the position of a cadaveric examination.
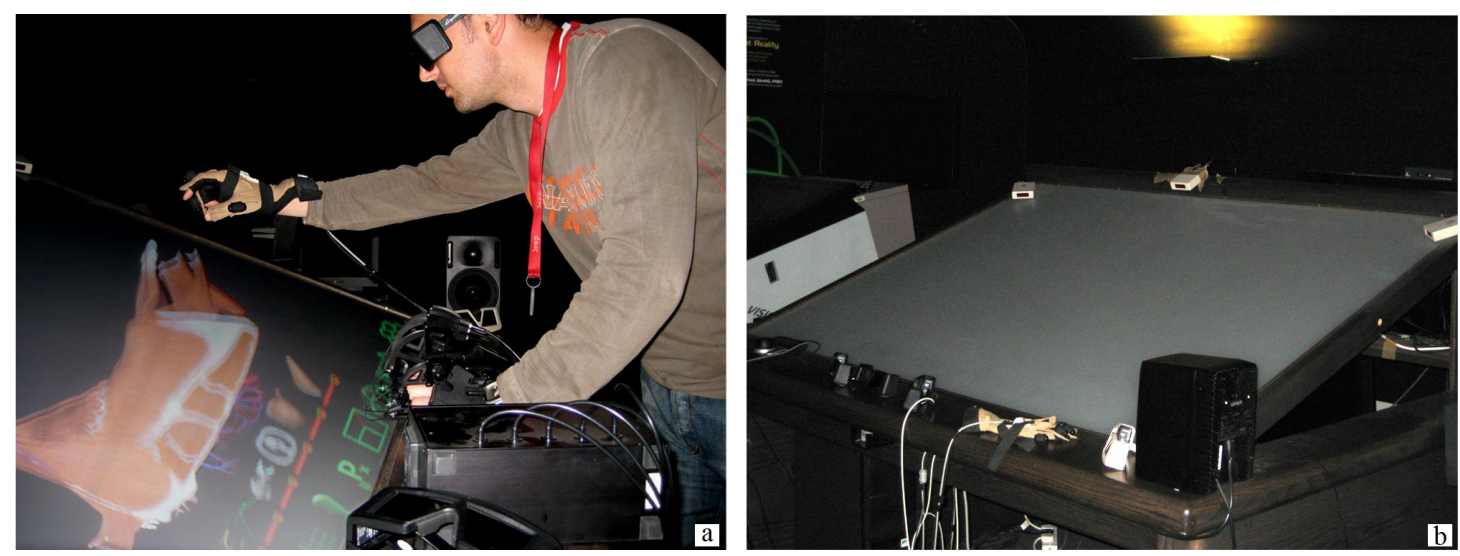

Figure 1: (a) Haptic interface demonstration for the manipulation of the VR human section

(b) The Fakespace Immersive Workbench with the CyberTouch ${ }^{\mathrm{TM}}$ sensored glove.

The virtual visualisation was achieved with CrystalEyes shutter glasses. We opted for this type of glasses as it was essential in this setting to provide the trainee and the trainer to see themselves and other members of the group as well as the model. In contrary fully immersive systems using head-mounted displays present a fully virtual environment which lacks the interactivity between the users during anatomy teaching or the trainee/trainer discussion of surgical rehearsal.

A minor pitfall of the system is that only one pair of glasses could be tracked by the table-sensors (typically the person using the haptic glove). Hence the rest of the viewers should be positioned as close as possible to that user (with the tracking-glasses) in order to have minimum visual distortion. However we intend to minimise the visual distortion experienced by the users with the non-tracking glasses by the development of a new VR facility with a substantially larger viewing surface.

The "physical" interaction between user and the VR model is enabled with the use of a tracked CyberTouch" ${ }^{\mathrm{TM}}$ sensored glove with vibro-tactile stimulators on the fingers and palm. This glove can sense the bend and relative position of the fingers and thumb, allowing interaction via gesture; 
combined with tracking it can sense the hand's position in space, allowing the user to explore and manipulate the digital model directly in 3D space.

Additionally 3D spatialised auditory cues provide sound effects which signalise a variety of different actions. The depth perception is extensively investigated with sound moves in space appropriate to the manipulation of the model. Notably the initial system was developed on the SGI platform. However contemporary CPUs, graphic card accelerators and large capacity memory DIMMS made implementable the transfer to a high-end PC platform. As such, the prospective system's cost efficiency was reduced radically offering the ability for the research team to experiment in more complex interfaces, models and even consider the development of a mobile system.

\section{CONTENT DEVELOPMENT}

\subsection{D Model Reconstruction}
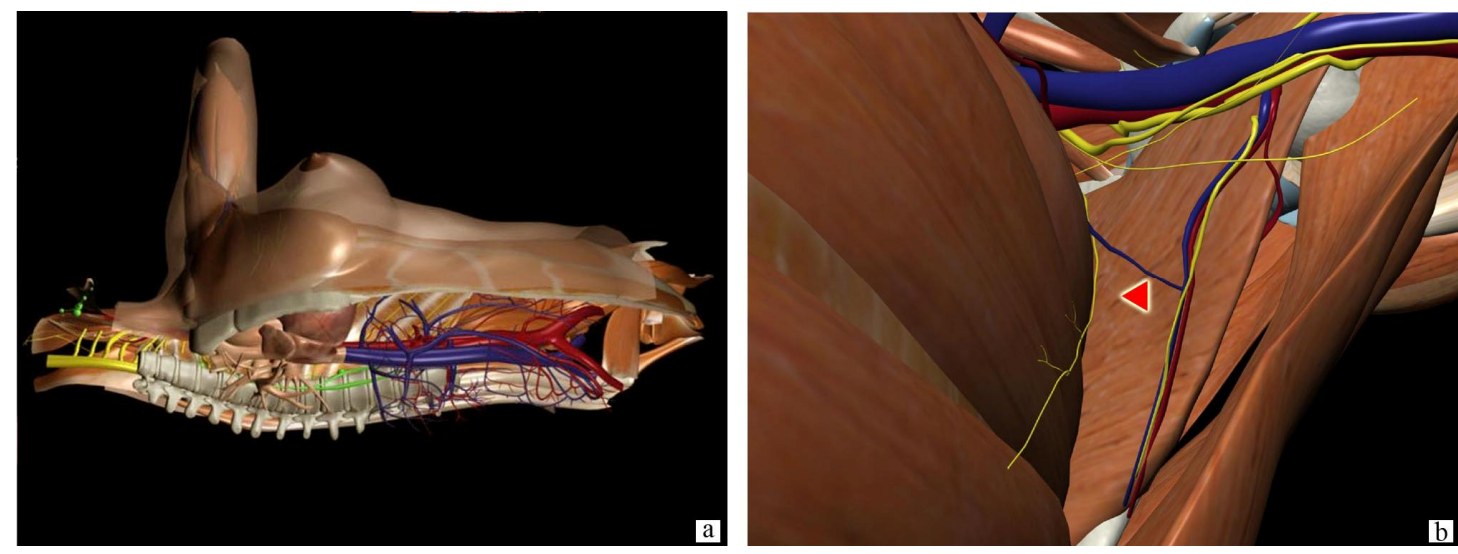

Figure 2: (a) Complete, detailed section of the female VR human body.

(b) Close-up of the 3D model with emphasis on the angular vein and inter-neural zone.

In order to evaluate the effectiveness of the proposed VR teaching method we developed a female 3D model under the guidance of Consultant Breast surgeons. Particular emphasis has been given to the axilla, its contents and surface markings. The nervous, venous, arterial and lymphatic systems were meticulously modelled to depict accurately the complex structures and their relationships as shown in figure 2. Notably the initial 3D human body data was developed with the use of CT and MRI scans. However the over-complex data had to be remodelled substantially in order to comply with the anatomical road-map required during the trainee test evaluation. The identical replication of details that present anatomical landmarks was of outmost importance for the successful conveyance of relevant information to the users. Additionally specific areas of the anatomy under investigation were elaborately developed with the constant input of information provided by the surgeons. Particularly the angular vein of the axilla, first described in 1993 [16]. This was introduced as it is a key surgical landmark in axillary clearance [17]. This exemplifies the importance of this collaborative methodology, and indeed the difference between taught and surgical operative anatomy.

In particular the three dimensional human model's complexity had to be restrained in order to comply with the maximum polygon-number computational ability provided by the customised personal computer. Therefore the model was further reformed and evaluated for optimum performance during projection and navigation. Interestingly the hierarchy and structure of the human model had to be adapted for the display and human computer interaction as well as for serving the examination process (i.e. questions sequence). 


\subsection{HCI Development}

The interaction between the user and the system was investigated explicitly in order to facilitate the requirements for the anatomy teaching of the particular area. Therefore the overall HCI was tailored to simplify the examination of the human model.

The interface interactivity was pushed beyond traditional information and data visualisation by including real-time transparencies in specific sections and organs of the human body enabling the users to comprehend the spatial relationship between lymphatic, vein, and musculoskeletal parts of the body. Interactivity means design parameters can be changed using the same user interface used for the visualisation. In the case of the product model, this means that the user may pick and change objects in real-time. Furthermore these customised interactivities could carry auxiliary functions which might include predetermined or real time simulations for different types of surgical rehearsal. The current virtual model exemplifies such an interactive scenario, as it highlights the potential problems during the surgical rehearsal for a malignant breast disease operation. As the specific interface is under development we form a tentative plan of work in order to incorporate extensive functionality and artificial intelligence in the system, so as to offer various guidelines or suggestions to the user either for the learning anatomy or for understanding the procedures for specific surgical operations.

Incorporating multiple viewing alternatives (i.e. predetermined positions of the specific section) and defining objects or structural parts that can be changed interactively (i.e. pectoralis major and minor transparency) required equally custom-tailored models as well as appropriate interface development that could support such functionalities. Hence the content of the experiment was heavily supported and highlighted from the intuitive HCI design. To this end we consider that an innovative HCI design may be composed of similar interface elements but their re-arrangement and context should be customised heavily for serving different anatomical issues and surgical rehearsal cases. Hence finding ways to make such issues easily understandable from different medical practitioners is illustrative of our approach.

\section{EXPERIMENT RATIONALE}

The validation study is based around contemporary experiments described in the literature and includes refinements drawn from other group's experiences. In particular it was decided to assess both the quantitative impact on learning outcomes and explore qualitatively the user's experience of the VR interface, their interaction with the data and the perceived educational value of the application. The study design is essentially a comparative randomised control trial with pre- and post-intervention assessment utilising matched quantitative and qualitative instruments.

\subsection{Subjects}

The target subjects of the validation comprised postgraduate doctors with a declared interest in surgery and one to two years of clinical experience in the National Health Service (NHS). Currently all graduating medical students must complete two years of organised generic postgraduate training (the Foundation Programme) prior to competitive selection for clinical specialities. As part of this process it is important that they complete not only in the foundation programme itself, but also undertake external collegiate membership (entrance) examinations in their chosen discipline. The targeted learning outcomes in the study came directly from the intercollegiate MRCS, and so focused directly on this group. To this end we approached Foundation Year doctors within Scotland and asked for volunteers for the validation study as 
depicted in figure 3 . The response rate was high, as many were studying for surgical membership and saw the study as a route to structured teaching time (total 36).

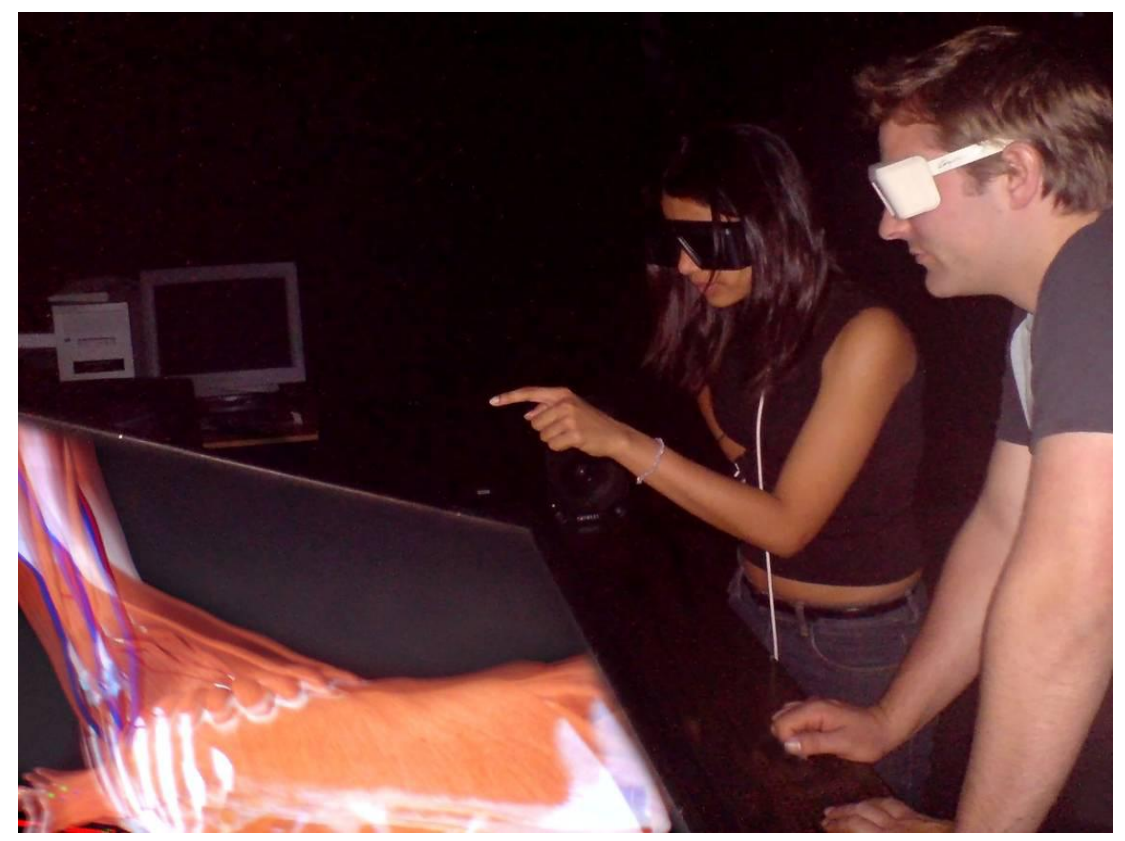

Figure 3: Actual screenshot during the evaluation

The volunteers were pre-assessed for anatomical knowledge and prior experience of 3D rotational applications. Twenty-three met the predetermined criteria and twenty were selected. This produced a balanced group of trainees in terms of graduating university (Edinburgh, Glasgow, Dundee, Aberdeen), sex, experience and age. These were then randomised to two trial arms.

The subjects were all current trainees and it is for this reason (ie: rota commitments) that the final total sample size was twelve rather than twenty. This lead to a rather small group size, but was sufficient for this scoping study. This illuminates a major problem for similar studies in this group, and has lead to the concept of developing a portable VR test-bed that can be taken to regional training days and thus increase future sample sizes.

\subsection{Experimental Methods}

The FY doctors were randomised into two groups:

1. Structured tutorial (traditional resources)

2. Structured tutorial (proposed VR system)

The groups then undertook their respective tutorials at the Digital Design Studio, Glasgow. Both groups were time limited to 25 minutes and guided by the instructor to a identical pre-determined protocols. The tutorial format was activity-based and situated, i.e.: designed to prompt the student into interaction with the real world/VR environment facilitated by the instructor.

The protocol was designed using operative expertise in collaboration with Consultant Breast Surgeons. The learning objectives included surgical anatomical knowledge, the principles of surgical management and operative treatment.

Attempts were made to control variables. All tutorials were undertaken in the same 2 month period the same with the identical protocol, model, equipment and instructor. The tutorials were 
repeated several times with the maximum of two subjects taking part at any time. Immediately after the tutorial all trainees were debriefed using identical instruments.

\subsubsection{Qualitative Method}

The subjects were debriefed using a forty point subjective user evaluation questionnaire. Additionally open questions were used to illuminate development issues and direct further work. The questionnaire used a nine point Likert scale, allowing a neutral position. The questions were grouped to explore the interface utility, interactivity, efficiency, subject engagement, and perceived spatial learning. Internal consistency was measured using Cronbach's alpha, responses were analysed by group and individual means.

\subsubsection{Quantitative Method}

Multiple Choice Questions (MCQs) were used to debrief all subjects. A set of twenty questions, both True/ False and common stem questions were used. The questions were structured into three groups. Respectively these explored spatial anatomy, the surgical approach and clinical principles. Each group was analysed finding total and question group means, standard deviations and confidence interval $(\mathrm{P}<0.05)$.

In this paper we were particularly interested in the way the FYs perceive the system's interactivity and how that facilitated contextual spatial learning. Additionally we sought quantative information in order to ensure quality measurement and assurance (i.e. explore the possibility that the system damages learning outcomes). As such the quantative results were reassuring in that they indicated that there was little overall difference between the MCQ scores in either group (VR goup mean 61.25 vs Traditional group mean 60.41).

\section{RESULTS}

\subsection{Thematic Analysis}

Two major themes pre-dominate the responses to the open questions. These themes support the findings of the user questionnaire. The first and most prevalent was exhibited in opposition between the groups and regarded engagement with the activity-based scenario. It is interesting to note that in the VR group students repeatedly commented on the 'immersion' and 'engagement' when speaking about their experience of the tutorial. One student said simply:

\section{'I found it really easy to get into it; it brings the anatomy to life, very like the dissecting lab'}

This was almost reversed in the traditional group, where several group members commented on the difficulty they experienced in 'getting into it", this is one example:

it reminded me of role playing, which I was never very good at as an undergraduate. '

It is interesting to note there were no complaints of difficulty engaging within the VR group.

The second most theme related to the subjects confidence in their understanding. It is quite telling that both groups felt the tutorial enhanced their learning of anatomy, but when asked about their confidence that they had learned accurately, a common response in the traditional group was that they were sometimes unsure if they had communicated their doubts clearly: 
'it was difficult. I was asked a question, and I don't know if we were talking about the same thing.'

In contrast members of the VR group found that communication with the trainer was enhanced and that individual misconceptions were exposed:

'If its in front of you, you can both see. He (the instructor) can see if you are wrong. '

It is interesting to note that both groups perceived the same tutorial in very different ways, despite the shared protocol. The results appear to indicate strongly that the ability to share the 3D experience of the anatomy between the trainer and trainee is important, as the analysis of the questionnaires depicts in figure 4.

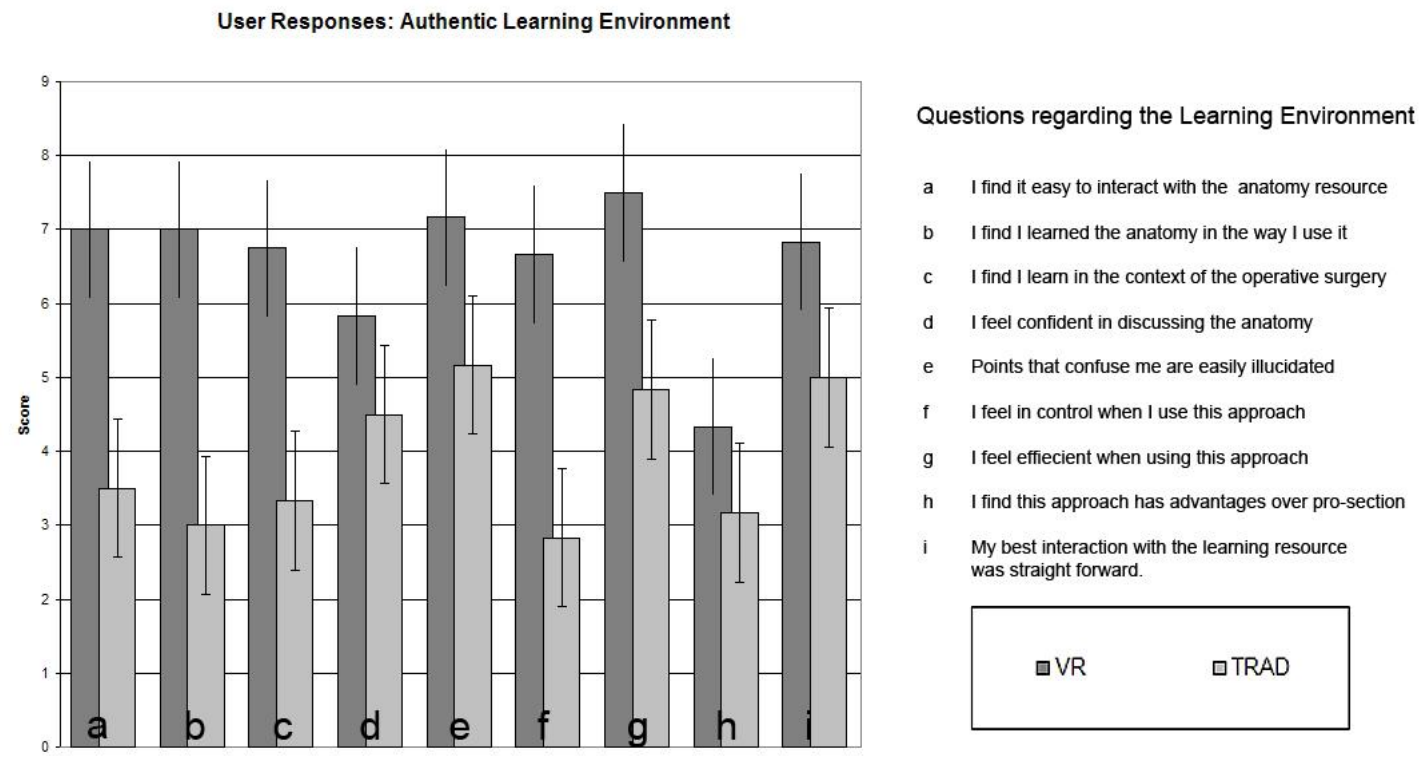

Figure 4: Comparative results between VR teaching environment and the traditional techniques.

\subsection{Qualitative Analysis}

The above results present a preliminary but informative assessment of how the VR learning environment augments contemporary teaching methods. Interestingly this small evaluation demonstrates the potential impact of a surgical accurate model displayed interactively via VR (figure 4).

Notably the use of the VR human model was preferred overall by the trainees in both groups. Specifically, the majority of participants found the VR anatomy considerably easier to interact with and understand as questions (a) and (b) shown respectively. Evidence collected also suggests that VR augmentation also engages and empowers the student (f). Additionally the results show that with the use of VR the student is not only a receiver of information but he/she is also enabled to interact in real-time with the given data. This reflects the advantages of situated, activity-based approaches. Additionally students in the VR group describe enhanced communication with their instructor (e). Also of note the students seem to express increased confidence in the accuracy of their knowledge $(\mathrm{g})$. 
Finally the results obtained from question (i) indicate that the functionalities of the human computer interaction (HCI) was accumulated very quickly. This can be attributed to the design of the interface as well as the familiarity of the contemporary students with complex operating systems throughout their daily life.

\section{CONCLUSIONS}

This paper describes the rationale and development process of a user-centred interface designed for a VR Medical training environment. The system was evaluated in a comparative study against the traditional anatomy teaching techniques. In this preliminary study we identified the several positive elements of VR augmentation and highlighted the potential pitfalls. The results suggest users perceived enhanced learning outcomes due to being able to investigate the human body without any physical constraint. However the complexity of the haptic interaction renders the system immobile and hence limits its use. To this end we currently investigate a mobile solution that incorporates "off the shelf" data-manipulating devices (i.e. Xbox or Playstation controllers) and we plan in the near future to repeat our user-tests with these devices and larger groups. Finally, our tentative plan for future work includes further development of the VR interface to include practical pre-operative surgical rehearsal for core surgical procedures.

\section{REFERENCES:}

[1] Sang-Hack, Jung \& Bajcsy, R., "Learning Physical Activities in Immersive Virtual Environments." In: IEEE Proceedings of the International Conference on Computer Vision Systems, ICVS '06, St. Johns University, Manhattan, New York City, USA, (2006).

[2] Henderson K., "On Line and On Paper, Visual Representations, Visual Culture, and Computer Graphics in Design Engineering", The MIT Press, Cambridge, Massachusetts, (1999).

[3] Nicolson T., Chalk C., "Can virtual reality improve anatomy education? A randomised controlled study of a computer-generated three-dimensional anatomical ear model", Medical Education 2006; 40: 1081-1087. (2006).

[4] Pandey, P., Zimitat, C., "Medical Students' Learning of Anatomy: Memorisation, Understanding and Visualization", In: Medical Education, Volume 41(1), Blackwell Science,(2007).

[5] Turney BW, "Anatomy in a modern medical curriculum", In: Annals of the Royal College of Surgeons of England. 89(2): 104-7, (2007),

[6] Ward B.M., Charissis V., Rowley D., Anderson P., and Brady L., "An Evaluation of Prototype VR Medical Training Environment: Applied Surgical Anatomy Training for Malignant Breast Disease", in Proceedings of the 16th International Conference of Medicine Meets Virtual Reality, Long Beach, California, USA. (2008).

[7] Ward B.M., Charissis V., Young I., Rowley D., and Garden O.J., "An Inquiry into the applications and Validation of Volumetric 3D Anatomy" , in Proceedings of the SARS International Conference, Birmingham, UK, (2008).

[8] Prensky, M., "Digital Game-Based Learning". Toronto: McGraw-Hill, (2001).

[9] Kneebone RL; Kidd J; Nestel D; Barnet A; Lo B; King R; Yang GZ; Brown R. "Blurring the boundaries: scenario-based simulation in a clinical setting". Medical Education 39:580-587. (2005).

[10] Shaffer, D. W., \& Resnick, M., “Thick authenticity: New media and authentic learning”, Journal of Interactive Learning Research, 10(2), 195-215, (1999).

[11] Charissis, V., Nomicos, S. and Patera, M., "Prototype System for Virtual Reality Simulation and Training: Initial Implementation for Large Printing Units", in Proceedings of the 3rd International Conference on Typography and Visual Communication (3rd ICTVC 07), From Verbal to Graphic, Thessaloniki, Greece, (2007).

[12] Patera, M. and Draper, S., "Immersive 3D visualisation and teaching colour use to interior designers", in Proceedings of the CAL'07 (Computer Assisted Learning) Development, Disruption $\&$ Debate, Trinity College, Dublin, Ireland, (2007). 
[13] A. Garg, G. Norman, L. Sperotable, "How medical students learn spatial anatomy", In The Lancet 2001, Elsevier, 363-364. (2001).

[14] Gay, G. and Hembrooke, H., "Activity-Centred Design: An Ecological Approach to Designing Smart tools and Usable Systems", The MIT Press, Cambridge, Massachusetts, USA, (2004).

[15] Anderson, P., Kenny, T. and Ibrahim, S., "The role of emerging visualisation technologies in delivering competitive market advantage", in Proceedings of the 2nd International Conference on Total Vehicle Technology, Institute of Mechanical Engineers, University of Sussex, Brighton, UK, pp.87-97, ISBN 186058377 6, (2002).

[16] O'Rourke MGE, Layt CWL., Angular vein of the axilla and the anatomy of the subscapular vein important in axillary node dissection. Aust N Z J Surg 1993; 63: 396-398, (1993).

[17]Y. Chan, M. Tan. Spatial relations of the angular vein, an important landmark in axillary nodal dissection, In: British Journal of Surgery, Volume 90, Issue 8, Pages 948-949. (2003) 